published in The Journal of Chemical Physics, J. Chem. Phys. 122, p. 054501 (2005), doi:10.1063/1.1839574

\title{
Breakdown of the mirror image symmetry in the optical absorption/emission spectra of oligo(para-phenylene)s
}

\author{
Georg Heime* \\ School of Chemistry and Biochemistry, Georgia Institute of Technology, Atlanta, Georgia 30332-0400 \\ Institute of Solid State Physics, Graz University of Technology, Petersgasse 16, A-8010 Graz, Austria \\ Maria Daghofer \\ Institute of Theoretical and Computational Physics, \\ Graz University of Technology, Petersgasse 16, A-8010 Graz, Austria \\ Johannes Gierschner \\ Institute of Physical and Theoretical Chemistry, University of Tübingen, \\ Auf der Morgenstelle 8, D-72076 Tübingen, Germany \\ Emil J. W. List \\ Christian Doppler Laboratory Advanced Functional Materials \\ Institute of Solid State Physics, Graz University of Technology, Petersgasse 16, A-8010 Graz, Austria \\ Institute of Nanostructured Materials and Photonics, Joanneum Research, A-8160 Weiz \\ Andrew C. Grimsdale and Klaus Müllen \\ Max-Planck-Institut für Polymerforschung, Ackermannweg 10, D-55128 Mainz, Germany \\ David Beljonne \\ Service de Chimie des Matériaux Nouveaux, Centre de Recherche en Electronique et Photonique Moléculaires, \\ Université de Mons-Hainaut, B-7000 Mons, Belgium \\ Jean-Luc Brédas \\ School of Chemistry and Biochemistry, Georgia Institute of Technology, Atlanta, Georgia 30332-0400 \\ Egbert Zojer \\ Institute of Solid State Physics, Graz University of Technology, Petersgasse 16, A-8010 Graz, Austria \\ School of Chemistry and Biochemistry, Georgia Institute of Technology, Atlanta, Georgia 30332-0400
}

(Dated: July 5, 2018)

\begin{abstract}
The absorption and emission spectra of most luminescent, $\pi$-conjugated, organic molecules are the mirror image of each other. In some cases, however, this symmetry is severely broken. In the present work, the asymmetry between the absorption and fluorescence spectra in molecular systems consisting of para-linked phenyl rings is studied. The vibronic structure of the emission and absorption bands is calculated from ab-initio quantum chemical methods and a subsequent, rigorous Franck-Condon treatment. Good agreement with experiment is achieved. A clear relation can be established between the strongly anharmonic double-well potential for the phenylene ring librations around the long molecular axis and the observed deviation from the mirror image symmetry. Consequences for related compounds and temperature dependent optical measurements are also discussed.

Keywords: ab-initio, electron-phonon coupling, absorption, emission, Franck-Condon, phenyl
\end{abstract}

\section{INTRODUCTION}

The poly(para-phenylene)s and their planarized derivatives are of particular interest in the field of $\pi$-conjugated materials due to their use in opto-electronic applications $\stackrel{1-6}{-6}$ The coupling between electronic and vibrational degrees of freedom strongly affects the nature of the photoexcitations in these materials. Thus, a good understanding of this coupling mechanism is highly desirable to tune the fundamental materials properties.

Because of strong electron-phonon coupling, organic $\pi$-conjugated molecules are subject to struc- tural distortions upon photoexcitation. Different BornOppenheimer potential energy surfaces in the ground state (GS) and electronically excited state (ES) lead to a reorganization of the molecular geometry in the ES in conjunction with an energetic stabilization. This strong electron-vibration coupling dramatically influences the bandshapes of the electronic transitions in absorption and emission, leading to a pronounced vibronic structure in the optical spectra. Usually, the major distortion upon photoexcitation can be attributed to a change in the $\mathrm{C}=\mathrm{C}$ bond alternation along the backbone. The related $\mathrm{C}=\mathrm{C}$ stretch vibrations lie in the energy range 
between $1200-1600 \mathrm{~cm}^{-1}$ and can be clearly resolved in (resonant) Raman spectra. ${ }^{7-12}$ The optical spectra exhibit an extended vibronic progression associated with these modes.

In a simple Franck-Condon (FC) picture, the absorption and emission spectra are expected to be the mirror images of each other. Experimentally, one observes mirror imaged absorption and emission spectra with a sharply resolved vibronic progression in molecules (e.g. oligoacenes $)^{13}$ and polymers (e.g. ladder-type poly (paraphenylene) $)^{14-16}$ that have a rigid backbone. For species that comprise torsional degrees of freedom in their conjugated backbone, varying degrees of deviation from the mirror image behavior can be observed. At room temperature, poly(para-phenylenevinylene) (PPV) and its oligomers exhibit a strong deviation from mirror image symmetry with the vibronic structure in the absorption spectrum being rather blurred and smeared out while the vibronic progression in the emission spectrum is relatively well-resolved ${ }^{17-19}$ At low temperature, however, the absorption spectrum gradually becomes more structured and mirror symmetry is recovered. ${ }^{20,21}$ Poly(para-phenylene) (PPP) and its oligomers ${ }^{22,23}$ as well as poly(fluorene) ${ }^{24-28}$ exhibit a broad, completely featureless and unstructured absorption band while in emission a clearly resolved vibronic progression appears. It is the purpose of this work to investigate this strong deviation from the mirror image symmetry for systems constituted of para-linked phenylene rings, addressing also the influence of temperature.

For PPV-type polymers and PPP, the broadening of the absorption has been explained by the presence of a distribution of site energies associated with different conjugation lengths ${ }^{29-34}$ As a consequence, one would observe a superposition of vibronically structured absorption spectra with different electronic origins, resulting in a featureless absorption band. Since exciton migration towards the lowest energy sites (longest conjugated segments) can take place prior to emission, fluorescence would then come mainly from a single species (the longest conjugated segments) with well-defined electronic transition energy. Thus, in emission, one would observe the sharply resolved vibronic progression of that species only.

Although this effect is very likely to occur in polymers, it might not necessarily be the dominant one for PPV and its oligomers $\frac{35}{2}$ In this case, the room-temperature blurring of the absorption spectra can be associated with the thermal activation of low-frequency librational modes. ${ }^{20}$ The approach pursued in the present study indicates that this explanation does not hold for PPP-related systems. In the latter, the strongly anharmonic potential for the ring librations can be held responsible for the dramatic breakdown of the mirror image symmetry in the absorption and emission spectra, as suggested in Ref. 36 .

In order to shed light on the influence of the low-energy torsional vibrations of the phenylene rings on the asymmetry between absorption and emission spectra, we investigated $p$-terphenyl $(3 \mathrm{P})$ and indenofluorene $(3 \mathrm{~F})^{37}$

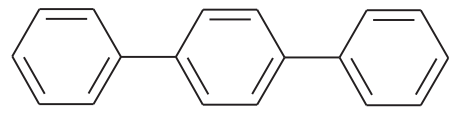

(a) $p$-terphenyl

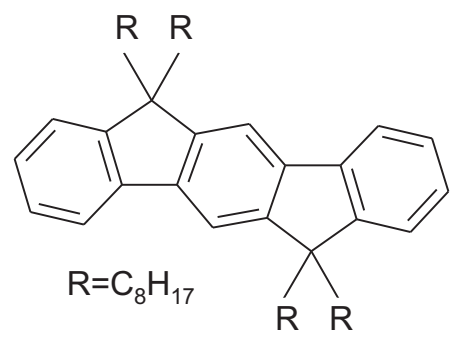

(b)indenofluorene

FIG. 1: Chemical structures of the model compounds $p$ terphenyl (a) and indenofluorene (b) investigated in this study.

(see Fig. 11). These systems are related to the more technologically relevant polymeric species, ladder-type poly(para-phenylene) and poly(fluorene) $\underline{\underline{1}-6}$ The electronic structure arising from the conjugated backbone is nearly identical for the two compounds. The important difference is that the phenylene rings are able to librate around the long molecular axis in $3 \mathrm{P}$, while the additional methyl bridges in $3 \mathrm{~F}$ keep the backbone rigidly planar and thus effectively block any torsional motion. ${ }^{38}$ The conjugation length is well-defined in oligomers, thereby leading to a narrower density of states with respect to polymers. Consequently, exciton migration phenomena can be expected to play a minor role for the shape of the optical spectra in oligomers. In addition, the experiments reported here have been conducted in dilute solution where energy transfer is slow compared to radiative decay.

The aim of this study is to show that a rigorous $\mathrm{FC}$ treatment of both high-energy $\mathrm{C}=\mathrm{C}$ stretching vibrations and low-energy ring librations in $3 \mathrm{P}$ can account for the breakdown of the mirror image symmetry in the case of $3 \mathrm{P}$ as opposed to $3 \mathrm{~F}$, where the mirror symmetry is retained. All required parameters are obtained from highlevel $a b$-initio calculations. The strongly anharmonic torsional potential in $3 \mathrm{P}$ is treated by numerically evaluating the librational energy levels, wavefunctions, and FC overlap integrals in curvilinear internal coordinates. Furthermore, we underline the importance of this procedure by comparing the results to a strictly harmonic approximation for the GS torsional potential well.

\section{THEORY}

Within the Born-Oppenheimer and Franck-Condon approximation, the probability $P_{g e}(E)$ for absorption of 
a photon with energy $E$ that promotes the molecule from the GS into its first ES can be split into an electronic part and a vibrational part:

$$
P_{g e}(E) \propto\left(\mu_{e l}^{0}\right)^{2} P_{\mathbf{v}^{\prime} \mathbf{v}^{\prime \prime}}(E)
$$

where the electric transition dipole matrix element $\left(\mu_{e l}^{0}\right)$ is evaluated at the ground-state equilibrium conformation of the molecule. The oscillator strength of the electronic transition is redistributed among all the transitions between the vibrational manifold of the ground and excited state. Assuming that the vibrational Hamiltonian of a molecule of $N$ nuclei can be separated into $3 N-6$ internal normal coordinates $Q_{i}$, the total vibrational wavefunction can be written as $\left|\mathbf{v}^{\prime}\right\rangle=\left|v_{1}^{\prime}\right\rangle\left|v_{2}^{\prime}\right\rangle \ldots\left|v_{3 N-6}^{\prime}\right\rangle$ and $\left|\mathbf{v}^{\prime \prime}\right\rangle=\left|v_{1}^{\prime \prime}\right\rangle\left|v_{2}^{\prime \prime}\right\rangle \ldots\left|v_{3 N-6}^{\prime \prime}\right\rangle$ for the ground and excited state, respectively. The $v_{i}^{\prime}$ and $v_{i}^{\prime \prime}$ are the vibrational quantum numbers of the individual oscillators, the total set of quantum numbers is denoted as $\mathbf{v}^{\prime}$ and $\mathbf{v}^{\prime \prime}$. The vibrational part of the transition probability, $P_{\mathbf{v}^{\prime} \mathbf{v}^{\prime \prime}}$, depends on the incoming photon energy $E$. Assuming a Gaussian broadening for all individual transitions, it can be written as:

$$
\begin{aligned}
P_{\mathbf{v}^{\prime} \mathbf{v}^{\prime \prime}}(E) & \propto \sum_{v_{1}^{\prime}}^{\infty} \ldots \sum_{v_{3 N-6}^{\prime}}^{\infty} \sum_{v_{1}^{\prime \prime}}^{\infty} \ldots \sum_{v_{3 N-6}^{\prime \prime}}^{\infty} B(T)\left|F_{\mathbf{v}^{\prime}}^{\mathbf{v}^{\prime \prime}}\right|^{2}(2) \\
& \times G\left(E ; \Gamma, E_{e l}+\sum_{i=1}^{3 N-6}\left(\varepsilon_{i}^{\prime \prime}\left(v_{i}^{\prime \prime}\right)-\varepsilon_{i}^{\prime}\left(v_{i}^{\prime}\right)\right)\right)
\end{aligned}
$$

$B(T)$ is a Boltzmann weighting factor that determines the population of the vibrational states within the manifold of the initial electronic state (GS for absorption, ES for emission) at finite temperature:

$$
B(T)= \begin{cases}\frac{1}{Z} \exp \left(-\frac{\sum_{i=1}^{3 N-6} \varepsilon_{i}^{\prime}\left(v_{i}^{\prime}\right)-\varepsilon_{i}^{\prime}(0)}{k_{B} T}\right) & \text { abs. } \\ \frac{1}{Z} \exp \left(-\frac{\sum_{i=1}^{3 N-6} \varepsilon_{i}^{\prime \prime}\left(v_{i}^{\prime \prime}\right)-\varepsilon_{i}^{\prime \prime}(0)}{k_{B} T}\right) & \mathrm{em} .\end{cases}
$$

where $Z$ is a normalization factor.

The $G$-Term on the far right hand side of Eq. 2 describes a normalized, Gaussian lineshape, which is a function of $E$, has a width (standard deviation) $\Gamma$, and is centered around the energy given by the third argument. $E_{e l}$ stands for the energy of the electronic origin (transition energy between the minima of the ground and excited state parabolas) and $\varepsilon_{i}^{\prime}$ is the energy eigenvalue of the $i^{\text {th }}$ oscillator along $Q_{i}^{\prime}$ containing $v_{i}^{\prime}$ quanta.

The term $F_{\mathbf{v}^{\prime}}^{\mathbf{v}^{\prime \prime}}$ in Eq. 2 describes the FC overlap between the total vibronic wavefunctions of the initial and final states:

$$
\begin{aligned}
F_{\mathbf{v}^{\prime}}^{\mathbf{v}^{\prime \prime}=} & \left\langle v_{1}^{\prime \prime}\left(Q_{1}^{\prime \prime}\right) \ldots v_{3 N-6}^{\prime \prime}\left(Q_{3 N-6}^{\prime \prime}\right)\right| \\
& \times\left|v_{1}^{\prime}\left(Q_{1}^{\prime}\right) \ldots v_{3 N-6}^{\prime}\left(Q_{3 N-6}^{\prime}\right)\right\rangle
\end{aligned}
$$

In the general case, the $Q_{i}^{\prime}$ in the GS differ from the set of $Q_{i}^{\prime \prime}$ in the ES. They are related by:

$$
\mathbf{Q}^{\prime}=\mathbb{D} \mathbf{Q}^{\prime \prime}+\Delta \mathbf{Q}^{\prime \prime}
$$

where $\mathbf{Q}^{\prime}$ and $\mathbf{Q}^{\prime \prime}$ abbreviate the complete set of $3 N-$ 6 internal normal coordinates $\left(Q_{i}^{\prime}\right.$ and $\left.Q_{i}^{\prime \prime}\right)$ in the GS and ES, respectively. $\Delta \mathbf{Q}^{\prime \prime}$ is the displacement between the equilibrium geometries in the electronic GS and ES expressed in the basis of the $Q_{i}^{\prime \prime}$ and the rotation between the GS and ES normal coordinates $\left(\mathbf{Q}^{\prime}\right.$ and $\left.\mathbf{Q}^{\prime \prime}\right)$ is given by $\mathbb{D}$, the Duschinsky matrix. If $\mathbb{D} \approx \mathbf{1}$ (which will be assumed in the present study), the multi-center integral in Eq. 4 can be factorized as:

$$
\begin{aligned}
\tilde{F}_{\mathbf{v}^{\prime}}^{\mathbf{v}^{\prime \prime}} & =\prod_{i=1}^{3 N-6}\left\langle v_{i}^{\prime \prime}\left(Q_{i}^{\prime \prime}\right) \mid v_{i}^{\prime}\left(Q_{i}^{\prime \prime}+\Delta Q_{i}^{\prime \prime}\right)\right\rangle= \\
& =\prod_{i=1}^{3 N-6}\left\langle v_{i}^{\prime \prime}\left(Q_{i}^{\prime \prime}-\Delta Q_{i}^{\prime \prime}\right) \mid v_{i}^{\prime}\left(Q_{i}^{\prime \prime}\right)\right\rangle
\end{aligned}
$$

In order to take partly into account the changes in interatomic force constants upon photoexcitation, two strategies are pursued: (i) The first is to project the geometry distortion between the GS and ES equilibrium geometries, $\Delta \mathbf{Q}$, onto the normal modes of the accepting electronic state ( $\Delta \mathbf{Q}^{\prime \prime}$ for absorption and $\Delta \mathbf{Q}^{\prime}$ for emission). (ii) Given that the Cartesian atomic displacements $X_{i}$ are related to the normal coordinates via $Q_{i}=\sqrt{\bar{m}_{i}} X_{i}$, with $\bar{m}_{i}$ being the effective mass of the $i^{t h}$ mode, the FC overlap matrix elements of Eq. [6 can be rewritten:

$$
\begin{aligned}
& F_{\mathbf{v}^{\prime}}^{\mathbf{v}^{\prime \prime}}=\prod_{i=1}^{3 N-6}\left\langle v_{i}^{\prime \prime}\left(Q_{i}^{\prime \prime}-\Delta Q_{i}^{\prime \prime}\right) \mid v_{i}^{\prime}\left(\sqrt{\bar{m}_{i}^{\prime}} X_{i}^{\prime \prime}\right)\right\rangle \\
& F_{\mathbf{v}^{\prime}}^{\mathbf{v}^{\prime \prime}}=\prod_{i=1}^{3 N-6}\left\langle v_{i}^{\prime}\left(Q_{i}^{\prime}+\Delta Q_{i}^{\prime}\right) \mid v_{i}^{\prime \prime}\left(\sqrt{\bar{m}_{i}^{\prime \prime}} X_{i}^{\prime}\right)\right\rangle
\end{aligned}
$$

where Eq. 7a holds for absorption and Eq. 7b for emission. For parabolic potentials, the vibronic wavefunctions $\left|v_{i}\right\rangle$ are known (harmonic oscillator wavefunctions) and the FC factors in Eq. 7 can be readily evaluated, allowing for different frequencies and different effective masses of GS and ES vibrational states. For general potentials, one needs to resort to numerical procedures (vide infra).

When calculating absorption spectra from Eq. 1, it needs to be taken into account that the absorption coefficient $\alpha(E)$ is related to the transition probability $P_{g e}(E)$ via:

$$
\alpha(E)=E P_{g e}(E)
$$

using the first lines of Eqs. 3 and 7 for the calculation of $P_{\mathbf{v}^{\prime} \mathbf{v}^{\prime \prime}}(E)$ (Eq. 2).

The transition probability for spontaneous emission, $P_{e g}(E)$, relates to $P_{g e}(E)$ as: $:^{39}$

$$
P_{e g}(E) \propto E^{3} P_{g e}(E)
$$

using the second lines of Eqs. 3 and 7 for the calculation of $P_{\mathbf{v}^{\prime} \mathbf{v}^{\prime \prime}}(E)$ (Eq. 2). 
Finally, if one detects fluorescence intensity $I_{e g}(E)$ per energy interval rather than the number of emitted particles, the measured signal is given by:

$$
I_{e g}(E)=E P_{e g}(E) \propto E^{4} P_{g e}(E)
$$

\section{METHODOLOGY}

\section{A. Experimental}

Absorption spectra of indenofluorene ${ }^{37}$ have been recorded with a Perkin-Elmer $\lambda-9$ UV/VIS/NIR spectrometer. Fluorescence spectra have been taken with a Shimadzu RF5301 spectrophotofluorometer that has been calibrated for spectral sensitivity with an Ocean Optics LS-1-CAL calibration light source. Measurements have been conducted at ambient temperature in dilute $(<1 \mu \mathrm{g} / \mathrm{ml}) \mathrm{CH}_{2} \mathrm{Cl}_{2}$ solution. Room temperature absorption and fluorescence spectra of $p$-terphenyl have been taken from Ref. 23.

For low temperature fluorescence measurements on $p$-terphenyl, it was dissolved in $n$-decane to obtain a solution with absorbance $\mathrm{E}=0.05$. The sample was placed in a $0.5 \mathrm{~cm}$ cylindrical quartz cell of Suprasil quality in a closed cycle He cryostat (Kryogenics Technology, model 501A) and measured on a Spex Fluorolog 222 spectrofluorimeter in backscattering configuration.

\section{B. Computational}

\section{Quantum chemical approach}

In order to obtain a reliable description of the geometric deformation taking place upon exciting the molecules from the GS into the ES, both electronic states have to be treated at a comparable level of theory. Therefore, GS energies, equilibrium geometries, normal coordinates, and vibrational frequencies were calculated within the Hartree-Fock (HF) approximation. ES energies, equilibrium geometries, normal coordinates, and vibrational frequencies were computed by employing a configuration interaction scheme on top of the HF calculation considering only singly excited Slater determinants (CIS) 40 The core orbitals were exempt from the variational CI procedure, all valence and virtual orbitals were included. A 6$311+\mathrm{G}(\mathrm{d}, \mathrm{p})$ basis set ${ }^{41-44}$ was used. For the subsequent calculation of absorption and emission bandshapes, the vibrational frequencies were scaled with a common factor of $0.9 . \stackrel{45}{ }$ All calculations were performed with GAUSSIAN98 ${ }^{46}$ using tight convergence criteria. The symmetry of both $3 \mathrm{~F}$ and $3 \mathrm{P}$ was constrained to $C_{2 h}$ throughout the calculations, forcing the individual phenylene rings to be strictly planar.

\section{Franck-Condon treatment}

For the high-energy, in-plane $\mathrm{C}=\mathrm{C}$ stretching vibrations of $3 \mathrm{~F}$ and $3 \mathrm{P}$, harmonic potentials and thus harmonic oscillator-type wavefunctions were assumed. The mass-weighted interstate distortion was projected onto the normal modes of the accepting electronic state in order to determine $\Delta Q_{i}$ (see Eqs. 5 to 7 ). The five most important modes (largest $\Delta Q_{i}$ ) were taken into account, covering more than $95 \%$ of the total interstate distortion. The first 5 vibrational states $\left(v_{i}=0 \ldots 4\right)$ of each mode were included in the calculation, yielding sufficiently converged results. All transitions with a FC weight of greater than $10^{-4}$ of the maximum weight transition have been evaluated. The width of the Gaussian bandshapes, $\Gamma$ (see Eq. 4), was set to $0.1 \mathrm{eV}$ in both molecules for better comparison with experiment.

\section{Torsional potentials}

As will be shown in the present study, the librational motion of the rings around the long molecular axis needs to be included for $3 \mathrm{P}$ in order to reproduce the experimentally observed bandshapes in absorption and emisssion. To that end, the diabatic torsional potentials in the electronic GS and ES of 3P have been computed on a $5^{\circ}$ grid in the interval $\left[-90^{\circ}, 90^{\circ}\right]$ assuming alternating signs for the inter-ring tilt angle. For the GS, we find a $W$-shaped, double-well potential with its minima at $\sim 45^{\circ}$ in accordance with results for biphenyl in gaseous phase. ${ }^{47.48}$ However, the experimental observations we seek to explain in this work have been obtained in solution, where the inter-ring tilt angle in $3 \mathrm{P}$ is experimentally found to be $\sim 30^{\circ}$ due to the hindrance imposed by the solvent molecules $\frac{49,50}{}$ in the form of an additional, $U$ shaped potential well. Furthermore, the rotational barrier at $0^{\circ}$ in solution is lower than that at $90^{\circ} \cdot 49,50$ In order to account for the influence of the solvent and the notorious overestimation of the central rotational barrier in $\mathrm{HF}, \underline{51}$ an additional single-well potential needs to be added to the shallow HF double-well potential (see Fig. 2). This additional, $U$-shaped potential cannot be derived from first-principles in a straightforward manner. As a tentative approximation, we chose to add $20 \%$ of the torsional potential in the ES to $80 \%$ of the original $\mathrm{HF}$ potential well of the GS.

The resulting double-well potential for the GS features a lower energy barrier at $0^{\circ}$ tilt angle $(\sim 0.19 \mathrm{eV})$ than at $90^{\circ}(\sim 0.36 \mathrm{eV})$ and the energy minima are pushed closer together $\left(\sim 35^{\circ}\right)$ towards the experimentally determined values.

We would like to point out that the main results of the present work are not affected by the exact shape of this additional, $U$-shaped torsional potential. Although quantitative agreement with experiment (e.g. Stokes shift) slightly depends on the specific choice for the correcting potential, any GS double-well deep enough to 


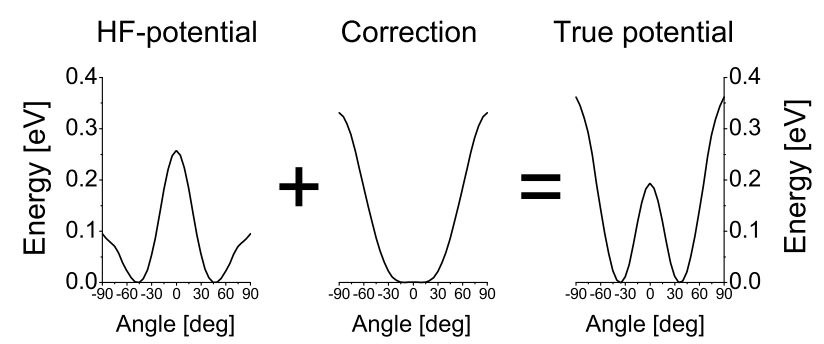

FIG. 2: Sketch of the process by which (in order to correct for methodological deficiencies and the influence of the solvent) a single-well potential (middle panel) has been added to the Hartree-Fock potential (left panel) in order to give the actual ground-state torsional potential for $p$-terphenyl (right panel). The energy scale is the same for all three graphs.

sustain librational states with an energy lower than the central rotational barrier leads to the same qualitative picture for the observed phenomena.

The ES torsional potential is $U$-shaped and almost parabolic. It exhibits much higher flanks than the GS potential, rendering it less susceptible to influences from the surroundings. Therefore, the ES torsional potential has been taken as produced by the CIS calculations.

\section{Numerical treatment of the libration}

In order to treat low-energy vibrations with an unusually large displacement $\Delta Q$ along the internal coordinate tilt angle $(\phi)$, one has to resort to curvilinear coordinates as opposed to linear Cartesian displacements in the case of the high-energy in-plane $\mathrm{C}=\mathrm{C}$ strech vibrations of the backbone. Failing to do so would result in strong but unphysical mode mixing of the torsion with in-plane stretch motions perpendicular to the long molecular axis $\underline{38,52}$ Furthermore, the GS torsional double-well potential in $3 \mathrm{P}$ is strongly anharmonic. It has been shown ${ }^{53-55}$ that anharmonic multiple-well potentials can lead to unconventional and strongly asymmetric vibronic progressions in soft conjugated molecules. Moreover, these authors suggested that a numerical evaluation of the vibrational wavefunctions and the FC overlap integrals leads to a better description of the experimental spectra.

The angular $(\phi)$ part of the librational Hamiltonian $H$ can be written (in cylindrical coordinates and adopting atomic units):

$$
H=\frac{1}{2 I} \frac{\partial^{2}}{\partial \phi^{2}}+W(\phi)
$$

The respective moments of inertia $I$ are extracted from the GS and ES equilibrium geometries. In order to solve the Schrödinger equation:

$$
H|v\rangle=\varepsilon|v\rangle
$$

the angle $\phi$ is discretized:

$$
\begin{aligned}
\phi \rightarrow \phi_{i} & =i \delta_{\phi} \\
i & =1,2, \ldots n \\
\delta_{\phi} & =\frac{\phi_{\max }}{n}
\end{aligned}
$$

where $\phi_{\max }=90^{\circ}$ in the particular case treated in the present work and $n=200$ was found to yield fully converged results. Subsequently, the librational wavefunction $|v\rangle$ and the potential need also to be defined on a discrete grid:

$$
\begin{aligned}
v(\phi) \rightarrow v_{i} & :=v\left(\phi_{i}\right) \\
W(\phi) \rightarrow W_{i} & :=W\left(\phi_{i}\right)
\end{aligned}
$$

The derivative in Eq. 11 is then expressed through finite differences:

$$
\frac{\partial^{2}}{\partial \phi^{2}} v(\phi) \rightarrow \frac{v_{i-1}-2 v_{i}+v_{i+1}}{\delta_{\phi}^{2}}
$$

Eventually, the Schrödinger equation can be written in a discrete manner:

$$
\frac{v_{i-1}-2 v_{i}+v_{i+1}}{\delta_{\phi}^{2}}+W_{i} v_{i}=\varepsilon v_{i}
$$

Taking into account periodic boundary conditions $\left(\phi_{n+1} \stackrel{!}{=} \phi_{1}\right)$, the resulting matrix can be diagonalized by standard algebraic methods (with eigenvalues $\varepsilon$ ).

In the present work, the lowest 30 energies and wavefunctions were calculated for both the GS and ES torsional potentials of 3P. Subsequently, the FC overlap integrals have been evaluated by numerical integration of the respective librational wavefunctions.

\section{RESULTS AND DISCUSSION}

\section{A. Experimental spectra}

The room-temperature experimental absorption and emission spectra of $3 \mathrm{~F}$ and $3 \mathrm{P}$ are shown in Figs. 3 and 4 For 3F, the mirror symmetry between absorption and emission is largely conserved. The deviations on the high energy side of the absorption spectrum are due to superimposed transitions to higher lying electronic states. Both emission and absorption exhibit a sharply resolved vibronic structure. In contrast, 3P features an entirely unstructured, broad absorption band as opposed to a much better resolved vibronic structure in emission. Moreover, the Stokes shift between the maxima of absorption and emission spectra in $3 \mathrm{P}(\sim 0.83 \mathrm{eV})$ is substantially larger than in $3 \mathrm{~F}(\sim 0.04 \mathrm{eV})$, suggesting a much larger reorganization energy in the ES. 


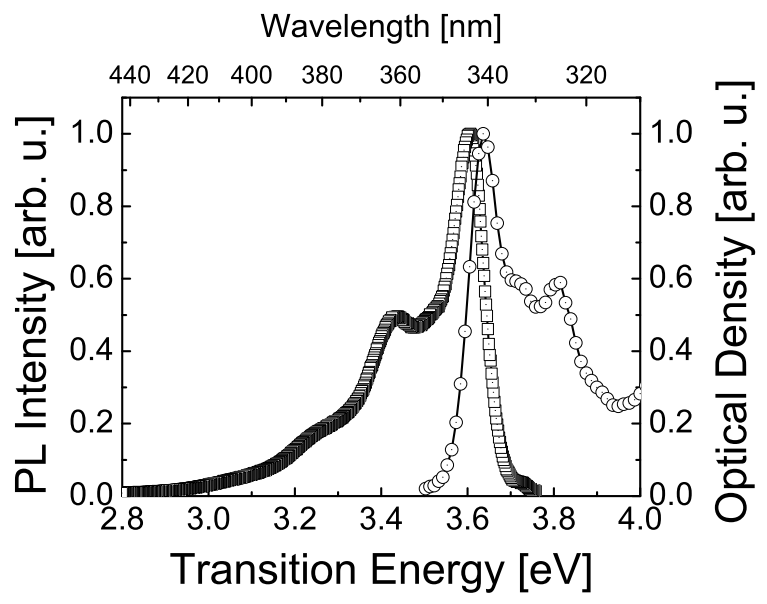

FIG. 3: Experimental absorption (circles) and emission (squares) spectra of indenofluorene in $\mathrm{CH}_{2} \mathrm{Cl}_{2}$ solution at ambient temperature.

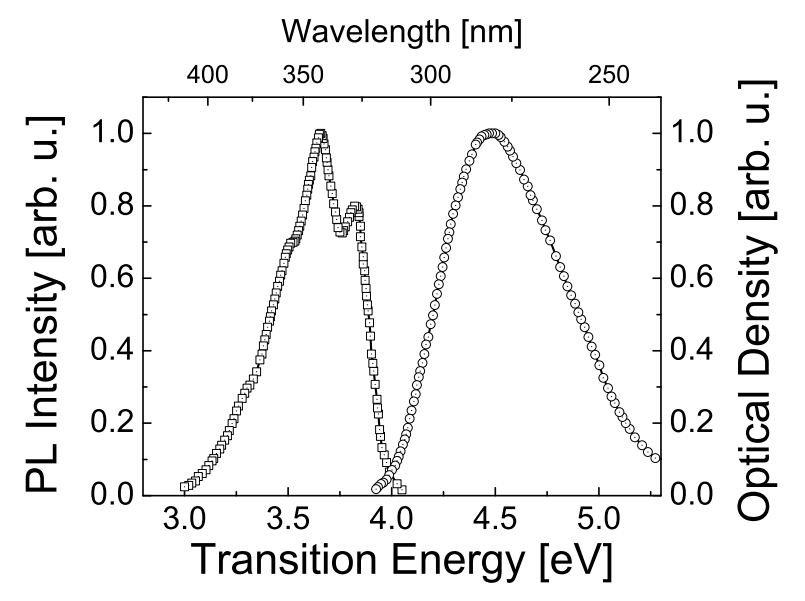

FIG. 4: Experimental absorption (circles) and emission (squares) spectra of $p$-terphenyl in cyclohexane solution at ambient temperature taken from Ref. 23.

\section{B. Calculated bandshapes}

\section{Indenofluorene}

Following the procedure outlined in Sections III and III. the major five contributing vibrational modes have been identified for 3F. The respective (unscaled) GS and ES frequencies and reduced masses are listed in Tab. [) Overall, the normal coordinates of GS and ES are found to coincide $\left(D_{i^{\prime} i^{\prime \prime}} \approx 1\right)$. The GS to ES Duschinsky rotation of mode no. 4 does not dramatically influence the outcome of the calculation since it has the smallest GS to ES distortion $\left(\Delta Q_{4}\right)$ associated with it. Inserting these
TABLE I: Unscaled vibrational frequencies $\omega_{i}\left(\right.$ in $\left.\mathrm{cm}^{-1}\right)$, effective masses $\bar{m}_{i}$ (in atomic mass units), and mass weighted projections $\Delta Q_{i}$ of the ground to excited state distortion (in $\sqrt{a m u} \AA$ ) of the five most important vibrations for indenofluorene. The respective Duschinsky matrix elements $D_{i^{\prime} i^{\prime \prime}}$ are also listed.

\begin{tabular}{crrrrrrrr}
\hline \hline \multirow{2}{*}{ Mode No. } & \multicolumn{3}{c}{ Ground State } & & \multicolumn{3}{c}{ Excited State } & \multirow{2}{*}{$D_{i^{\prime} i^{\prime \prime}}$} \\
\cline { 2 - 4 } \cline { 6 - 8 } & $\omega_{i}^{\prime}$ & $\bar{m}_{i}^{\prime}$ & $\Delta Q_{i}^{\prime}$ & & $\omega_{i}^{\prime \prime}$ & $\bar{m}_{i}^{\prime \prime}$ & $\Delta Q_{i}^{\prime \prime}$ & \\
\hline 1 & 221 & 5.49 & 0.43 & & 217 & 5.51 & -0.42 & 0.99 \\
2 & 555 & 4.29 & -0.11 & & 542 & 4.61 & 0.13 & 0.98 \\
3 & 821 & 6.09 & 0.17 & & 784 & 6.11 & -0.18 & 0.99 \\
4 & 1455 & 2.02 & -0.10 & & 1473 & 2.77 & 0.12 & 0.65 \\
5 & 1807 & 6.93 & 0.12 & & 1786 & 6.66 & -0.15 & 0.81 \\
\hline \hline
\end{tabular}

results into Eq. 7 allows us to evaluate the $\mathrm{FC}$ weights of all transitions between the vibrational manifolds of the GS and the ES. The resulting calculated bandshapes (Eq. 2) for absorption and emission at $295 \mathrm{~K}$ are shown in Fig. 5. The electronic origin has been set to the experimental value of $3.62 \mathrm{eV}$. The Stokes shift between the maxima of the two spectra (calculated to be $\sim 0.04 \mathrm{eV}$ ) is a direct result of the calculation. We find good overall agreement with the measured spectra shown in Fig. 3. Comparison with experiment indicates that the inhomogeneous line broadening is smaller in $3 \mathrm{~F}$ than it is in 3P. Reducing the damping factor in the calculated spectra of $3 \mathrm{~F}$ reveals more details in the vibronic structure (e.g., the shoulders at $\sim 3.5 \mathrm{eV}$ and $\sim 3.7 \mathrm{eV}$ in Fig. 3), all of which are fully consistent with the experimental results. However, a broadening of $0.1 \mathrm{eV}$ has been assumed in the calculations in order to stay consistent with the results presented for $3 \mathrm{P}$. The slight overestimation of the intensity of the 0-1 transition in absorption can be at-

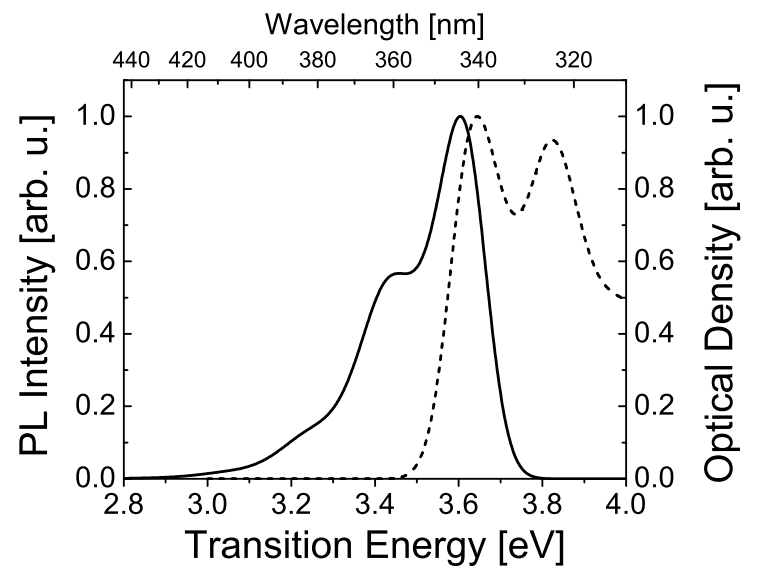

FIG. 5: Calculated spectral bandshapes for absorption (dashed line) and emission (solid line) of indenofluorene (3F). 
tributed to the (partial) neglect of Duschinsky rotation and/or the inaccuracy $(\sim 10 \%)$ of CIS force constants.

The most important result is that both absorption and emission spectra exhibit a sharply resolved vibronic progression. In accordance with experiment, the two spectra obey mirror image symmetry to a large extent.

\section{2. "Planar" p-terphenyl}

Applying the same computational procedure to 3P, one can again identify the five in-plane high-frequency modes that couple most strongly to the optical transition. Their respective (unscaled) GS and ES frequencies and reduced masses are listed in Tab. II]

TABLE II: Unscaled vibrational frequencies $\omega_{i}\left(\right.$ in $\left.\mathrm{cm}^{-1}\right)$, effective masses $\bar{m}_{i}$ (in atomic mass units), and mass weighted projections $\Delta Q_{i}$ of the ground to excited state distortion (in $\sqrt{a m u} \AA)$ on the five most important, high-energy, harmonic backbone vibrations for $p$-terphenyl. The respective Duschinsky matrix elements $D_{i^{\prime} i^{\prime \prime}}$ are also listed.

\begin{tabular}{|c|c|c|c|c|c|c|c|}
\hline \multirow{2}{*}{ Mode No. } & \multicolumn{3}{|c|}{ Ground State } & \multicolumn{3}{|c|}{ Excited State } & \multirow{2}{*}{$D_{i^{\prime} i^{\prime \prime}}$} \\
\hline & $\omega_{i}^{\prime}$ & $\bar{m}_{i}^{\prime}$ & $\Delta Q_{i}^{\prime}$ & $\omega_{i}^{\prime \prime}$ & $\bar{m}_{i}^{\prime \prime}$ & $\Delta Q_{i}^{\prime \prime}$ & \\
\hline 1 & 224 & 6.72 & -0.13 & 243 & 6.93 & 0.13 & 1.00 \\
\hline 2 & 838 & 5.99 & 0.04 & 811 & 6.15 & -0.11 & 0.83 \\
\hline 3 & 1301 & 1.14 & 0.16 & 1359 & 1.16 & -0.20 & 0.90 \\
\hline 4 & 1391 & 4.43 & 0.16 & 1460 & 4.47 & -0.15 & 0.98 \\
\hline 5 & 1804 & 5.62 & 0.18 & 1795 & 5.17 & -0.17 & 0.97 \\
\hline
\end{tabular}

In this first approach, the ring torsional vibrations are completely neglected. The respective calculated bandshapes for absorption and emission are presented in Fig. 6 for $295 \mathrm{~K}$ with the electronic origin set to the experimentally determined value of $3.99 \mathrm{eV}$.

The spectra in Fig. 6 nicely obey the mirror image rule and show a clearly resolved vibronic structure both in emission and in absorption. They do not, however, reproduce the experimental findings shown in Fig. 4.

\section{P-terphenyl in the harmonic approximation}

As opposed to $3 \mathrm{~F}$, which features a rigid backbone, the individual phenylene rings of $3 \mathrm{P}$ are free to librate around the long molecular axis. In the electronic GS, the competition between steric hindrance of the orthohydrogens and conjugation which tends to planarize the molecule leads to a double-well, $W$-shaped potential for the ring torsional motion. $\underline{56}$ Due to changes in the electronic structure of $3 \mathrm{P}$ upon photoexcitation, the interring bonds are strengthened in the electronic ES, thus overcoming the steric hindrance imposed by the orthohydrogens. Overall, this leads to a planar structure in the ES stabilized by an (almost) parabolic, $U$-shaped torsional potential. As a consequence of the substantial

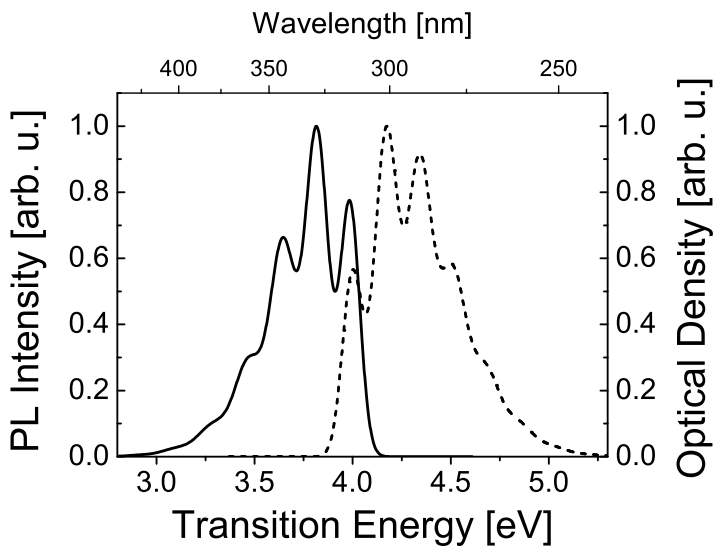

FIG. 6: Calculated spectral bandshapes for absorption (dashed line) and emission (solid line) in p-terphenyl (3P). The phenyl ring librations are not taken into account.

change of the inter-ring tilt angle between the equilibrium conformations in the GS and the ES, the librational mode strongly couples to the optical transition.

In order to demonstrate the importance of a proper consideration of the actual shape of the torsional poten-

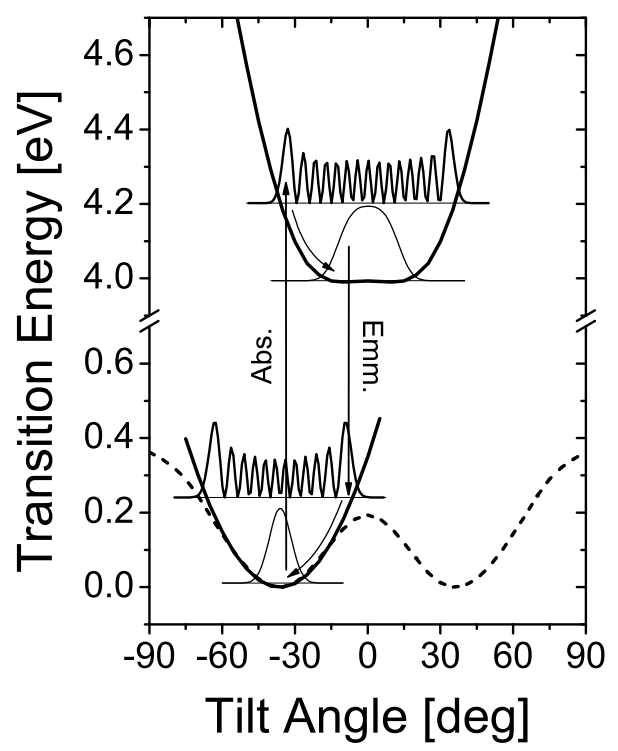

FIG. 7: The potential energy curves associated with the libration of the phenyl rings in the electronic ground (GS) and excited state of $p$-terphenyl (3P). The true GS potential is represented by the dashed curve, the harmonic approximation is solid. Probability amplitudes are plotted for the initial (thin solid line) and most important final state (thick solid line) for absorption and emission (within harmonic approximation). 
tial, we first report the results obtained when adopting the commonly applied harmonic approximation for the GS torsional potential well. ${ }^{38}$ To this end, one branch of the torsional double-well is approximated by a parabola centered at one of the double-well's minima (see Fig. 7). For reasons of consistency, the ES potential has been taken as calculated. Subsequently, the numerically procedure outlined in Subsection ПB has been applied in order to evaluate the transition energies and FC overlap matrix elements.

The situation sketched in Fig. 7 is (almost) symmetrical for absorption and emission. Given the large GS to ES displacement $(\Delta Q=\sqrt{I} \Delta \phi)$ associated with the torsional vibration, one would expect a far extended vibronic progression of finely separated lines with (almost) Poisson distributed intensities $20,35,38$ In order to illustrate the contribution to the total bandshapes coming from the ring torsional vibration within the harmonic approximation to the GS potential well, the associated vibronic progression is plotted in Fig. 8 for $0 \mathrm{~K}$. As indicated in Figs. 7 and 8 , the most important accepting librational states have high quantum numbers of $v^{\prime}=11$ for emission and $v^{\prime \prime}=14$ for absorption. The latter value is close to the $\tilde{v}^{\prime \prime}=11$ reported for biphenyl in supersonic jet expansion spectroscopy 55

The energetic splitting of $100-170 \mathrm{~cm}^{-1}$ (see Ref. 55) between the individual transitions within the subset of the librational levels is too small to be resolved experimentally in solution. Because each of the well-resolved peaks stemming from the 5 high-energy harmonic backbone modes (see Fig. 6) is replaced by the respective librational progression (Fig. 8), this results in additional, almost Gaussian broadening. Due to the higher curva-

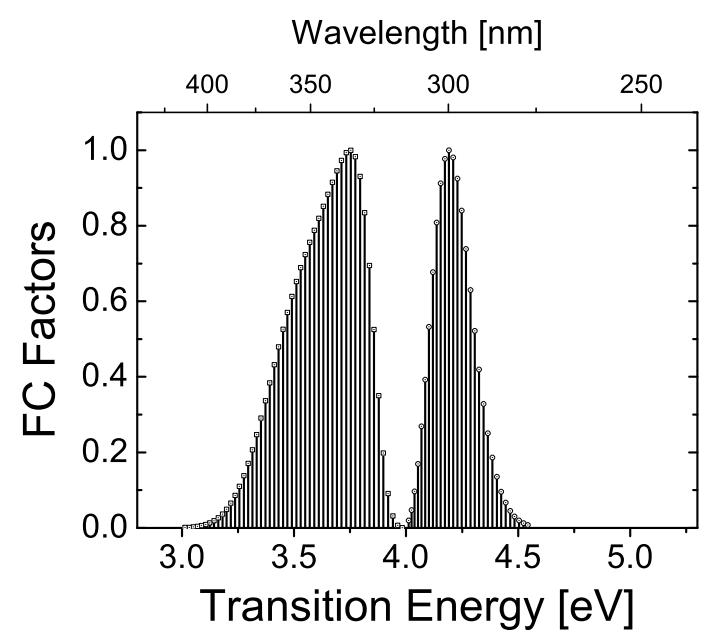

FIG. 8: Vibronic progression associated with the phenyl ring librations in $p$-terphenyl $(3 \mathrm{P})$ within the harmonic approximation for the torsional potential of the electronic ground state at $0 \mathrm{~K}$.

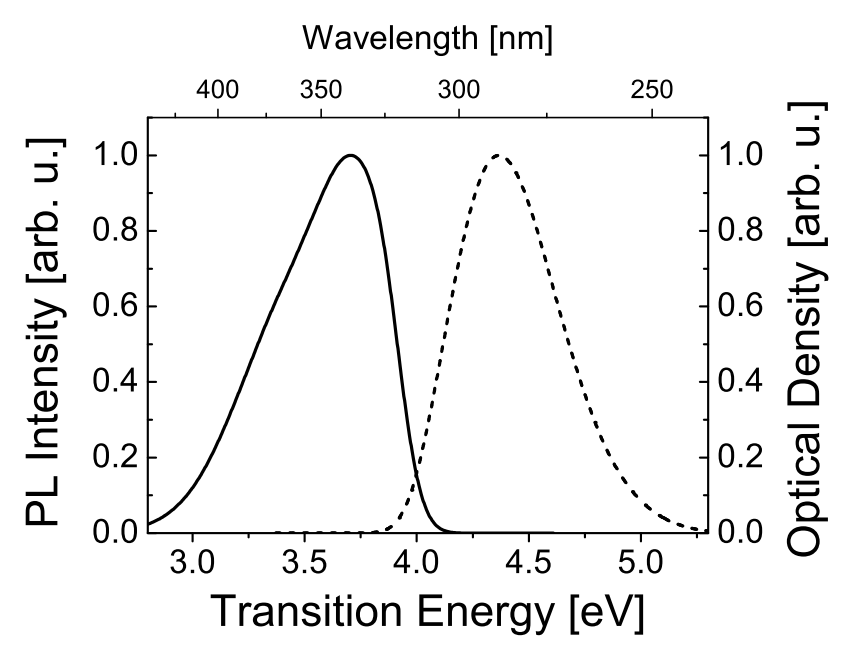

FIG. 9: Calculated spectral bandshapes for absorption (dashed line) and emission (solid line) in $p$-terphenyl (3P). The phenyl ring librations have been taken into account using the harmonic approximation for the torsional potential in the electronic ground state.

ture of the approximate (harmonic) GS torsional potential (wider spacing of levels) compared to the ES potential, this broadening affects the emission spectrum even more severely than the absorption.

The calculated bandshapes that take into account both the 5 high-energy modes (Tab. II) and the inter-ring libration in its harmonic approximation are shown in Fig. 9. In this case, both the emission and absorption spectra are equally smeared out to featureless bands, exhibiting a large Stokes shift, as has been predicted in Ref. 35. They do, however, obey mirror image symmetry which is in clear contradiction to the experimental observations (Fig. 4).

\section{Anharmonic torsion in p-terphenyl}

Only the inclusion of the actual, strongly anharmonic double-well potential for the GS torsion can account for the breakdown of the mirror image symmetry in oligo(para-phenylene)s in general and in $3 \mathrm{P}$ in particular, as pointed out in Ref. 36. The massive deviation of the GS potential from the harmonic approximation introduces the necessary asymmetry between the accepting librational states in GS and ES. The nearly harmonic ES potential features harmonic oscillator-like vibrational wavefunctions (see Figs. 10 and 7), which results in a wide vibronic progression associated with the ring torsion that smears out the absorption spectrum to an unstructured, broad band. In contrast, the strongly anharmonic potential in the GS leads to wavefunctions that deviate significantly from harmonic oscillator wavefunc- 


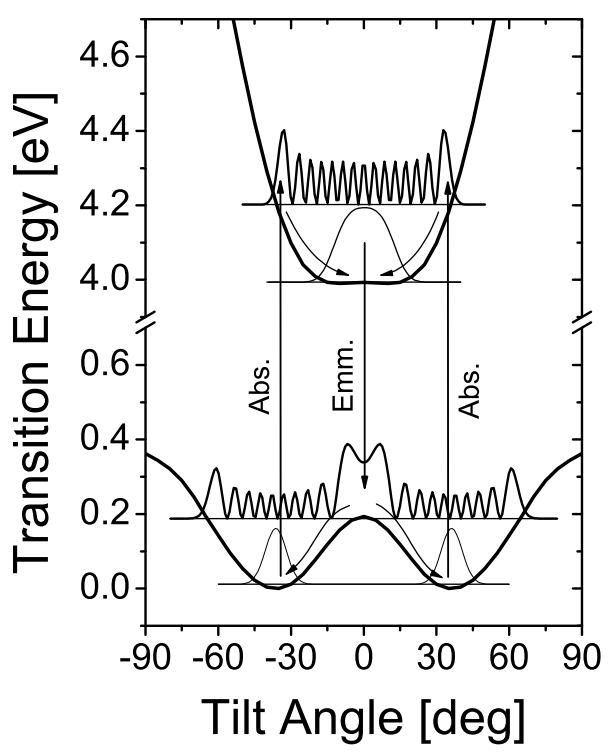

FIG. 10: The actual potential energy curves associated with the libration of the phenyl rings in the electronic ground (GS) and excited state of $p$-terphenyl (3P). Probability amplitudes are plotted for the initial (thin solid line) and most important final state (thick solid line) for absorption and emission.

tions (compare Figs. 10 and 7). This leads to a massive deviation of the associated vibronic progression from the Poisson-type envelop for emission.

To illustrate this feature, the progressions related to the phenyl ring librations are plotted in Fig. 11 for absorption and emission at $0 \mathrm{~K}$, taking full account of the actual GS torsional potential. The librational progression for absorption from the actual GS potential equals that from the harmonic approximation. The dominant accepting state is also that with $v^{\prime \prime}=14$. For emission, the most important accepting libronic state in the GS has a quantum number of $v^{\prime}=9$ and energetically lies just above the central potential barrier, thus exhibiting a probability maximum at $0^{\circ}$. Despite its high quantum number, the functional form of the associated wavefunction is somehow reminiscent of that of a harmonic oscillator ground-state function for a (harmonic) potential centered around $0^{\circ}$. This explains also why the overlap with the $v^{\prime \prime}=0$ librational wavefunction in the ES (also shown in Fig. (10) is largest. Effectively, the situation for emission resembles a system in which the libration does not couple at all to the optical transition (i.e., one in which the GS to ES interstate distortion along the associated normal coordinate is zero). This is reflected by the very narrow libronic progression for emission centered around the dominant $v^{\prime \prime}=0 \rightarrow v^{\prime}=9$ peak.

To describe the optical spectra, again each of the wellresolved peaks stemming from the 5 high-energy harmonic backbone modes (see Fig. 6) is replaced by the re-

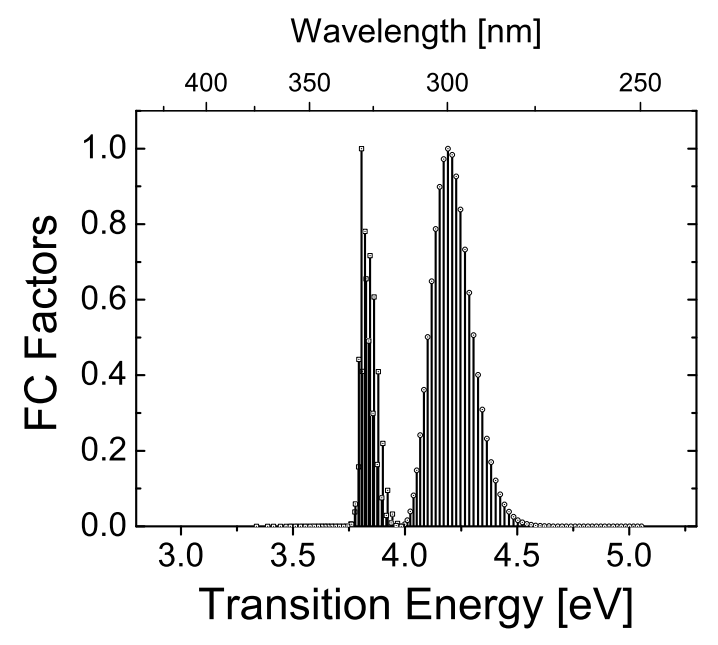

FIG. 11: Vibronic progression associated with the phenyl ring librations in $p$-terphenyl (3P) for the actual, librational double-well potential in the electronic ground state at $0 \mathrm{~K}$.

spective librational progression (Fig. 11). Taking the actual GS torsional potential into account, the sharp transitions get smeared out in absorption a lot more than in emission. This is a direct consequence of the combination of the (anharmonic) GS double-well with the (harmonic) ES single well, regardless of their exact shape.

Combining the results shown in Fig 11 with those presented in Fig. 6 yields the total bandshapes for absorp-

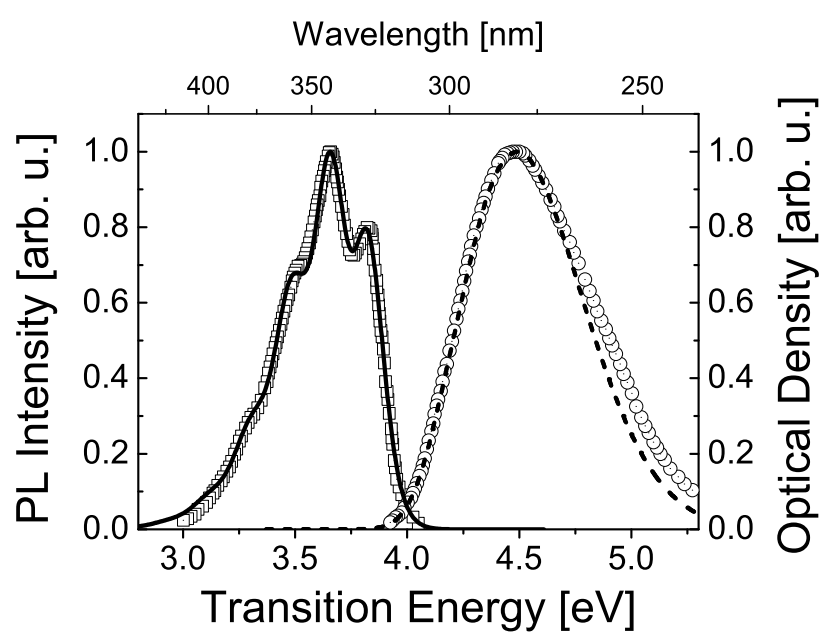

FIG. 12: Calculated absorption (dashed line) and emission (solid line) bandshapes for $p$-terphenyl taking into account the anharmonic double-well potential for the phenyl ring librations. The experimental absorption and emission spectra are represented by circles and squares, respectively. 
tion and emission in 3P. These are plotted in Fig. 12 together with the experimental data from Fig. 4. Here again, only the electronic origin has been shifted to the experimentally determined value of $3.99 \mathrm{eV}$, whereas the Stokes shift (calculated to be $\sim 0.83 \mathrm{eV}$ ) is a direct result of our calculations.

Comparing the optical spectra of $3 \mathrm{~F}$ and $3 \mathrm{P}$ shows that the Stokes shift is substantially larger in the latter. This implies that the reorganization energy in the excited state and thus the additional stabilization of the exciton is strongly increased by the presence of strongly coupled low-energy conformational degrees of freedom. This is fully consistent with the results of our calculations, which yield a reorganization energy of $0.34 \mathrm{eV}$ in $3 \mathrm{~F}$ and 2.5 times as much, $0.84 \mathrm{eV}$, for $3 \mathrm{P}$.

\section{RELATED SYSTEMS AND LOW-TEMPERATURE OPTICAL SPECTRA}

In solution, not only $3 \mathrm{P}$ but also all other oligo(paraphenylene)s (from biphenyl to $p$-sexiphenyl) exhibit the same broad, featureless absorption band as opposed to a vibronically well-structured emission. 23 Apart from the fact that starting with $p$-quaterphenyl there are more than one symmetric ring torsional vibration present in the system, we suggest that the mechanism outlined in the present work can account for the observed breakdown of the mirror image symmetry in all oligo(paraphenylene)s.

In contrast to PPV oligomers (vide infra), the observed asymmetry between the absorption and emission spectra in $3 \mathrm{P}$ is conserved also at low temperatures (see Fig. 13). This further supports our conclusion that the breakdown

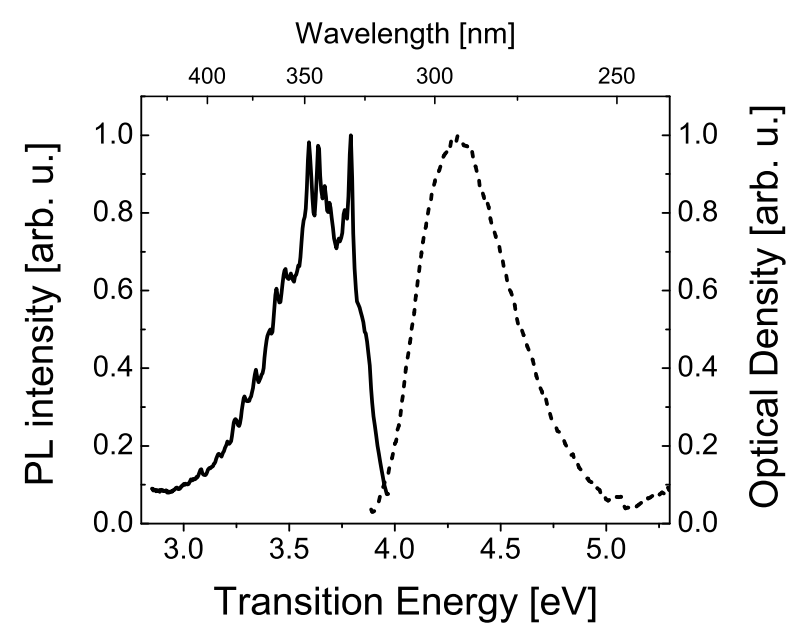

FIG. 13: Experimental absorption (dashed line) and emission (solid line) spectra of $p$-terphenyl in $n$-decane solution recorded at $15 \mathrm{~K}$. of the mirror image symmetry in $3 \mathrm{P}$ is indeed an intrinsic effect that is only related to the particular combination of the GS ( $W$-shaped) and ES ( $U$-shaped) torsional potentials and not to different thermal broadening of absorption and emission (we have not included calculated spectra for low temperature since the torsional potential wells used to calculate the spectral bandshapes in solution cannot be expected to correctly describe the rather complex situation 57 at low temperatures in solidified solution/matrix).

As mentioned in the introduction, $\mathrm{PPP}^{22}$ and poly(fluorene) ${ }^{24-28}$ also exhibit one broad unstructured absorption band and a well-resolved vibronic progression in emission, both in solution and in the solid state. Given the similarity of the chemical structures (phenylene rings para-linked by a C-C single bond), the double-well potential in the GS for the ring libration can be expected to prevail here. The same reasoning as for $3 \mathrm{P}$ can then be applied to these polymeric systems. Of course, effects related to excitation energy migration also play a role in polymeric samples with a broad distribution of effective conjugation lengths, as discussed in the introduction.

Temperature dependent optical studies on thin films of poly(9,9-(di- $n, n$-octyl)fluorene) revealed that in the so-called $\beta$-phase, the side chains crystallize, rigidly confining the polymer backbone to a planar conformation ${ }^{28,58-61}$ Librations of the individual repeat units are thus effectively hindered. As a consequence, the absorption spectrum associated with the $\beta$-phase exhibits a well-pronounced vibronic structure associated with high-energy, in-plane $\mathrm{C}=\mathrm{C}$ stretch modes, similar to that observed in emission. $25-28$

A number of temperature dependent optical measurements have also been performed on (unsubstituted) PPV oligomers in (frozen) solution and matrix ${ }^{20,21,62-65}$ The overall finding was that broadening of the backbone mode progressions significantly decreases upon decreasing temperature. The absorption spectrum evolves from a broad, relatively featureless band towards the mirror image of the sharply structured emission. The strong broadening of the absorption as compared to emission at ambient temperature has been attributed to thermal population of the librational levels (energy spacing wider in the ES than in the GS $)^{20}$. The PPV oligomers can be distinguished from the oligo(para-phenylene)s in that the former show a planar equilibrium geometry in the GS together with a high torsional flexibility, 20 whereas the latter exhibit a non-planar GS equilibrium geometry. Due to this qualitatively different form of the torsional potential wells in PPV oligomers (both harmonic singlewells centered at $0^{\circ}$, only different curvature in GS and ES), the slightly more involved approach pursued in this work is not necessary (though applicable) to satisfactorily model their optical spectra (compare Ref. 20). Experimentally, the difference between these two types of molecules (PPV and oligo(para-phenylene)s) cannot be told from the room-temperature optical spectra; it shows very clearly, however, at low temperature. 


\section{CONCLUSION}

By comparing the soft backbone $p$-terphenyl with the rigidly planar but otherwise identical indenofluorene, the violation of the mirror image symmetry in the optical spectra of $p$-terphenyl has been investigated. Applying ab-initio quantum chemical methods and further numerical treatment, the vibronically resolved bandshapes of the absorption and emission spectra of the investigated compounds have been calculated. The contribution of the phenylene ring librations in $3 \mathrm{P}$ has been simulated by numerical evaluation of the related wavefuntions and Franck-Condon factors in internal curvilinear coordinates, taking fully into account the substantial anharmonicity of the related potential wells.

Good agreement with experiment was achieved. A clear correlation between the torsional degree of freedom and the breakdown of mirror image symmetry could be established. It has been shown that only a rigorous Franck-Condon treatment that fully takes into account the strongly anharmonic double-well potential for the phenyl ring librations in $p$-terphenyl reproduces the experimental results. Furthermore, the failure of the harmonic approximation for shallow, massively anharmonic potential wells has been addressed.

Temperature dependent optical measurements on related compounds have been discussed following the lines of thought of the present work.

\section{Acknowledgments}

The authors would like to acknowledge the financial support of the SFB Elektroaktive Stoffe and project P14237-PHY of the Austrian Fonds zur Förderung der Wissnschaftlichen Forschung (FWF). The work at Georgia Tech is partly supported by the US National Science Foundation (CHE-034342). The work in Mons and in Tübingen was supported by the European Commission through the Human Potential Programme (RTN 'Nanochannel', Contract No. HPRN-CT-200200323). The work in Mons was also partly supported by the Belgian National Fund for Scientific Research (FNRS/FRFC) and by the Belgian Federal Government in the framework of the "Pôle d'attraction Interuniversitaire en Chimie Supramoléculaire et Catalyse Supramoléculaire (PAI5/3)". CDL-AFM is an important part of the long term AT\&S research activities.
* Electronic address: georg.heimel@chemistry.gatech.edu

1 C. Kallinger, M. Hilmer, A. Haugeneder, M. Perner, W. Spirkl, U. Lemmer, J. Feldmann, U. Scherf, K. Mllen, A. Gombert, et al., Adv. Mater. 10, 920 (1998).

2 G. Grem, G. Leditzky, B. Ulrich, and G. Leising, Adv. Mater. 4, 36 (1992).

3 A. W. Grice, D. D. C. Bradley, M. T. Bernius, M. Inbasekarian, W. W. Wu, and E. P. Woo, Appl. Phys. Lett 73, 629 (1998).

4 M. R. Craig, M. M. de Kok, J. W. Hofstraat, A. P. H. J. Schenning, and E. W. Meijer, J. Mater. Chem. 13, 2861 (2003).

5 M. Gross, D. C. Müller, H.-G. Mothofer, U. Scherf, D. Neher, C. Bräuchle, and K. Meerholz, Nature 405, 661 (2000).

6 M. T. Bernius, M. Inbasekaran, J. O'Brien, and W. Wu, Adv. Mater 12, 1737 (2000).

7 H. Ohtsuka, Y. Furukawa, and M. Tasumi, Spectrochim. Acta, Part A 49, 731 (1993).

8 S. Krichene, J. P. Buisson, and S. Lefrant, Synth. Met. 17, 589 (1987).

9 A. M. A. da Costa, N. Karger, A. M. Amado, and M. Becucci, Solid State Ion. 97, 115 (1997).

10 G. Louarn, L. Athouël, G. Froyer, J. P. Buisson, and S. Lefrant, Synth. Met. 57, 4762 (1993).

11 L. Cuff and M. Kertesz, Macromol. 27, 762 (1994).

12 L. Cuff, M. Kertesz, U. Scherf, and K. . Müllen, Synth. Met. 69, 683 (1994).

13 N. Nijegorodov, V. Ramachandran, and D. P. Winkoun, Spectrochim. Acta, Part A 53, 1813 (1997).

14 J. Stampfl, W. Graupner, G. Leising, and U. Scherf, J. Lumin. 63, 117 (1995).

15 W. Graupner, S. Eder, M. Mauri, G. Leising, and
U. Scherf, Synth. Met. 69, 419 (1995).

16 B. Schweitzer, G. Wegmann, D. Hertel, R. F. Mahrt, H. Bässler, F. Uckert, U. Scherf, and K. Müllen, Phys. Rev. B 59, 4112 (1999).

17 B. Tian, G. Zerbi, R. Schenk, and K. Müllen, J. Chem. Phys. 95, 3191 (1991).

18 L. Chiavarone, M. D. Terlizzi, G. Scamarcio, F. Babudri, G. M. Farinola, and F. Naso, Appl. Phys. Lett. 75, 2053 (1999).

19 E. Mulazzi, A. Ripamonti, J. Very, B. Dulieu, E. Faulques, and S. Lefrant, Synth. Met. 101, 196 (1999).

20 J. Gierschner, H.-G. Mack, L. Lüer, and D. Oelkrug, J. Chem. Phys. 116, 8596 (2002).

21 E. Peeters, A. M. Ramos, S. C. J. Meskers, and R. A. J. Janssen, J. Chem. Phys. 112, 9445 (2000).

22 G. Leising, T. Verdon, G. Louarn, and S. Lefrant, Synth. Met. 41-43, 279 (1991).

23 N. I. Nijegorodov, W. S. Downey, and M. B. Danailov, Spectrochim. Acta, Part A 56, 783 (2000).

24 E. Zojer, A. Pogantsch, E. Hennebicq, D. Beljonne, J. L. Brédas, P. S. de Freitas, U. Scherf, and E. J. W. List, J. Chem. Phys. 117, 6794 (2002).

25 E. K. Miller, G. S. Maskel, C. Y. Yang, and A. J. Heeger, Phys. Rev. B 60, 8028 (1999).

26 A. J. Cadby, P. A. Lane, H. Mellor, S. J. Martin, M. grell, C. Giebler, D. D. C. Bradley, M. Wohlgenannt, C. An, and Z. V. Vardeny, Phys. Rev. B 62, 15604 (2000).

27 M. Ariu, D. G. Lidzey, M. Sims, A. J. Cadby, P. A. Lane, and D. D. C. Bradley, J. Phys.: Condens. Matter 14, 9975 (2002).

${ }^{28}$ U. Scherf and E. J. W. List, Adv. Mater. 14, 477 (2002).

29 U. Rauscher, H. Bässler, D. D. C. Bradley, and M. Hennecke, Phys. Rev. B 42, 9830 (1990). 
30 J. Yu, J. H. Hsu, K. R. Chuang, C. L. Chao, S. A. Chen, F. J. Kao, W. S. Fann, and S. H. Lin, Synth. Met. 74, 7 (1995).

31 J. Yu and S. H. Lin, Synth. Met. 85, 1115 (1997).

32 J. Yu, M. Hayashi, S. H. Lin, K.-K. Liang, J. H. Hsu, W. S. Fann, C.-I. Chao, K. R. Chuang, and S.-A. Chen, Synth. Met. 82, 159 (1996).

33 E. Mulazzi, A. Ripamonti, L. Athouël, J. Wery, and S. Lefrant, Phys. Rev. B 65, 0852041 (2002).

34 E. Mulazzi, A. Ripamonti, J. Wery, B. Dulieu, and S. Lefrant, Phys. Rev. B 60, 16519 (1999).

35 S. Karabunarliev, M. Baumgartner, E. R. Bittner, and K. Müllen, J. Chem. Phys. 113, 11372 (2000).

${ }^{36}$ F. Momicchioli, M. C. Bruni, and I. Baraldi, J. Phys. Chem. 76, 3983 (1972).

37 J. Grimme and U. Scherf, Macromol. Chem. Phys. 197, 2297 (1996).

38 S. Karabunarliev, E. R. Bittner, and M. Baumgarten, J. Chem. Phys. 114, 5863 (2001).

39 G. C. Schatz and M. A. Ratner, Quantum Mechanics in Chemistry (Dover, Mineola, New York, 2002), p. 91.

40 J. B. Foresman, M. Head-Gordon, J. A. Pople, and M. J. Frisch, J. Phys. Chem. 96, 135 (1992).

41 M. J. Frisch and J. A. Pople, J. Chem. Phys. 80, 3265 (1984).

42 R. Krishnan, J. S. Binkley, R. Seeger, and J. A. Pople, J. Chem. Phys. 72, 650 (1980).

43 D. Feller, J. Chem. Phys. 96, 6104 (1992).

44 T. Clark, J. Chandrasekhar, G. W. Spitznagel, and P. von Ragué Schleyer, J. Comp. Chem. 4, 294 (1983).

45 J. B. Foresman and A. Frisch, Exploring Chemistry with Electronic Structure Methods (Gaussian, Inc., Pittsburgh, PA, USA, 1996), 2nd ed.

46 M. J. Frisch, G. W. Trucks, H. B. Schlegl, G. E. Scuseria, M. A. Robb, J. R. Cheeseman, V. G. Zakrzewski, J. A. Montgomery, Jr., R. E. Stratmann, et al., Gaussian 98, Rev. A.11, Gaussian, Inc., Pittsburgh PA (2001).
47 O. Bastiansen, Acta Chem. Scand. 3, 408 (1949).

48 A. Almenningen, O. Bastiansen, L. Fernholt, B. N. Cyvin, S. J. Cyvin, and S. Samdal, J. Mol. Struct. 128, 59 (1985).

49 M. Akiyama, Spectrochim. Acta, Part A 40, 367 (1984).

50 A. Ghanem, L. Bokobza, C. Noel, and B. Marchon, J. Mol. Struct. 159, 47 (1987).

51 S. Tsuzuki, T. Uchimaru, K. Matsumura, M. Mikami, and K. Tanabe, J. Chem. Phys. 110, 2858 (1999).

52 J. R. Reimers, J. Chem. Phys. 115, 9103 (2001).

53 M. Gouterman, J. Chem. Phys. 42, 351 (1965).

54 A. V. Naumov, N. L. Naumova, and I. S. Osad'ko, Opt. Spectrosc. 91, 704 (2001).

55 H.-S. Im and E. R. Bernstein, J. Chem. Phys. 88, 7337 (1988).

${ }^{56}$ H. Cailleau, J. L. Baudour, J. Meinnel, A. Dworkin, F. Moussa, and C. M. E. Zeyen, Faraday Discuss. Chem. Soc. 69, 7 (1980).

57 G. Swiatkowski, R. Menzel, and W. Rapp, J. Lumin. 37, 183 (1987).

58 M. J. Winokur, J. Slinker, and D. L. Huber, Phys. Rev. B 67, 1841061 (2003).

59 M. Ariu, M. D. Rahn, J. Hill, A. M. Fox, D. G. Lidzey, M. Oda, J. Cabanillas-Gonzalez, and D. D. C. Bradley, Phys. Rev. B 67, 1953331 (2003).

60 M. Grell, D. D. C. Bradley, G. Ungar, J. Hill, and K. S. Whitehead, Macromol. 32, 5810 (1999).

61 D. Neher, Macromol. Rapid Comm. 22, 1365 (2001).

${ }^{62}$ Y. Ichino, J. P. Ni, Y. Ueda, and D. K. Wang, Synth. Met. 116, 223 (2001).

63 O. Narwark, A. Gerhard, S. C. J. Meskers, S. Brocke, E. Thorn-Csányi, and H. Bässler, Chem. Phys. 294, 17 (2003).

64 T. W. Hagler, K. Pakbaz, K. F. Voss, and A. J. Heeger, Phys. Rev. B 44, 8652 (1991).

65 O. Narwark, S. C. J. Meskers, R. Peetz, E. Thorn-Csányi, and H. Bässler, Chem. Phys. 294, 1 (2003). 